\title{
AXIAL DISPERSION IN GASES FLOWING THROUGH A PACKED BED AT ELEVATED PRESSURES
}

\author{
A.H. BENNEKER, A.E. KRONBERG, J.W. POST, A.G.J. VAN DER HAM and \\ K.R. WESTERTERP*
}

Chemical Reaction Engineering Laboratories, Department of Chemical Engineering,

University of Twente, PO Box 217, 7500 AE Enschede, The Netheriands

\begin{abstract}
Axial dispersion in upward gas flow is investigated by pulse and displacement experiments in a vertical, packed column with different concentrations of the tracer and at pressures up to $1.5 \mathrm{MPa}$. The responses to the introduced pulse and step changes are measured at two locations and the extent of axial dispersion, represented by the Bodenstein number, is determined by curve fitting in the time domain. The performed experiments demonstrate that the residence time distribution is considerably affected by density differences between the tracer and carrier gas, particularly at elevated pressures. Obtained Bodenstein numbers for step changes from nitrogen to a helium/nitrogen mixture and vice versa differ by as much as a factor ten, depending on the helium concentration and column pressure. The difference in axial dispersion may be ascribed to gravitation-driven instabilities as due to vertical density gradients in the case of a heavy gas displaced by a light gas; density gradients in the step changes from a light to heavy gas evidently inhibit axial dispersion. The presented observations are of major importance for the description of flow behaviour of gases in packed bed reactors where density gradients exist due to temperature and concentration gradients, particularly because many processes operate at elevated pressures.
\end{abstract}

\section{INTRODUCTION}

Dispersion in the longitudinal direction affects the conversion and the selectivity in reactors and the separation effectiveness in many chemical apparatus. Therefore, for modelling, design and optimization of different types of equipment, e.g. adsorbers and catalytic reactors, knowledge of axial dispersion in packed beds is of major importance. Because it is not possible to predict directly or even to describe the hydrodynamic behaviour of fluid flow through packed beds, the extent of axial dispersion has to be determined experimentally by residence time distribution (RTD) measurements. The mixing data of a fluid flowing through a packed bed are usually given in the form of the Bodenstein number $B o=u d_{p} / \varepsilon D_{a x}$ which in its turn is related to the Reynolds number $R e=\rho u d_{p} / \varepsilon \eta$ or to a Bodenstein number based on the molecular diffusity $B o_{m o l}=u d_{n} / \varepsilon D_{n u i l}$. In spite of the large amount of experimental data now available on axial dispersion in packed beds, there is presently no general correlation which may be used with confidence to predict the Bodenstein number in packed beds. Roughly it has been observed that for gases $B o$ reaches a value of around 2 for $R e$ values above 10 and remains constant, although for beds packed with fine particles essentially lower Bodenstein numbers are reported, see f.i. Moulijn and Van Swaaij (1976), Langer et al. (1978) and Kehinde et al. (1983). Kehinde et al. (1983) also demonstrated for low Reynolds numbers that lower values of axial dispersion coefficients are obtained when other processes as interphase mass transfer and internal diffusion are present, whereas Chao and Hoelscher (1966) have found axial dispersion coefficients under conditions of interphase mass transfer to be significant higher than those measured under pure mixing conditions at higher Reynolds numbers. For liquids, $B o \approx 0.5$ for $R e<50$ and then increases slowly to reach a value of $B o \approx 2$ for $R e$ around 1000. Experiments with liquid flow through regular packings showed deviating mixing behaviour. It depends on the type of packing whether the Bodenstein number increases or decreases when the transition from laminar to turbulent flow occurs, as shown by Hiby (1962). Gunn and Pryce (1969) concluded that neither the mixing cell model nor the axially dispersed plug flow model could describe their dispersion phenomena in regular packings.

Explanations of experimental results by different authors are quite different, e.g. compare the analysis of the same mechanism of axial mixing by Levich et al. (1967), Gunn (1969, 1993) and Tsotsas and Schlünder (1988, 1994); many of the observed differences in mixing behaviour are still unexplained. The difference between gases and liquids is often ascribed to the difference in the Schmidt number $S c=\eta / \rho D_{m o l}$, see a.o. Gunn (1969) and Tsotsas and Schlünder (1988). However, for the regime $B o_{\text {mot }} \gg 1$ the molecular diffusion hardly plays a role in practical systems, and therefore Westerterp and Wijngaarden (1990) concluded that there must be another factor affecting the axial dispersion, which was confirmed by their experiments on axial and radial heat transfer in gases at different pressures. They developed a hypothesis that - besides the ratio of the kinetic energy and viscous dissipation - the fluid elasticity might be the unidentified governing factor, that influences the dispersion in packed beds. Recently,

\footnotetext{
"To whom correspondence should be addressed.
} 


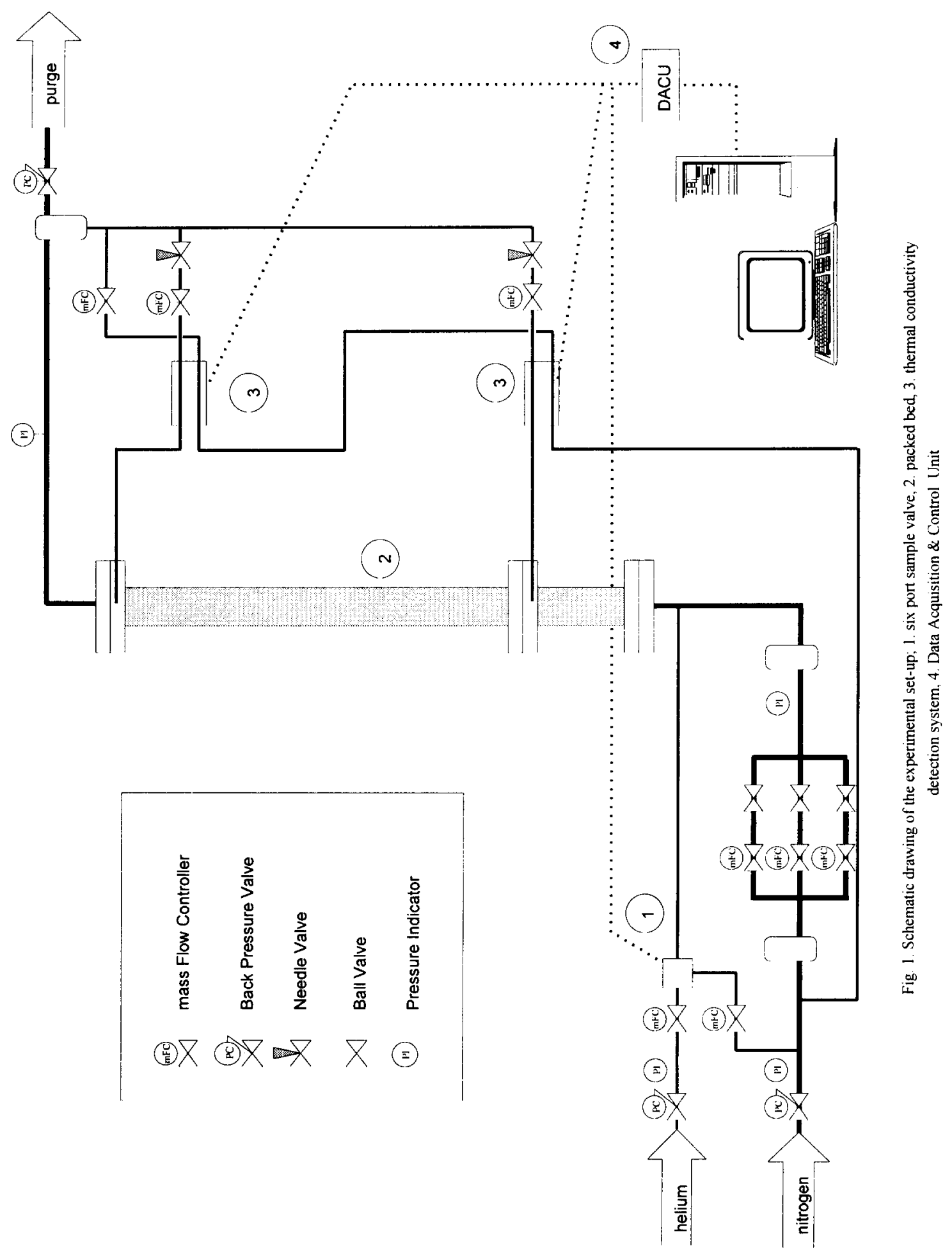


Westerterp et al. (1995b) have confirmed by their experiments on mass dispersion at elevated pressures there is an additional factor which influences the axial dispersion; the experimental Bodenstein numbers can not be presented as an unique function of the Reynolds and Schmidt numbers at different pressures.

It is recognized that density and viscosity differences give rise to significant changes in the nature of dispersion. even for fluids with a small variation of density and viscosity, see Nunge and Gill (1969). However, axial dispersion data are assumed to be determined at conditions without any influence of the tracer on the flow behaviour. Therefore many authors did not consider the influence of the tracer; others took measures to avoid this influence by doing experiments at a tracer concentration as small as possible limited only by its analytical detection. The experimental results obtained with a perfect tracer are, of course, important from the scientific point of view for understanding the flow behaviour of uniform fluids, but they can not be used with confidence for the description of most real chemical or physical processes. For the description of such processes, knowledge of mixing in the longitudinal direction is most important when compositions and/or temperatures are rapidly changing with respect to the time or axial coordinate, e.g. due to chemical reaction, interphase heat and/or mass transfer or transient operation. In consequence of such changes gradients in density and viscosity might exist and the influence of these on the axial mixing might be considerable. Hill (1952) probably was the first who paid attention to these phenomena in porous media. He measured an influence of density and viscosity differences during the refining of sugar, where a sugar liquor is displaced by water in vertical cisterns filled with granular charcoal. Such phenomena are now well-known in geothermal energy systems and in oil recovery processes, where the crude oil in underground reservoirs is displaced by a nonaqueous, miscible fluid, see the review of Nunge and Gill (1969). Displacement experiments with aqueous solutions of $\mathrm{KCl}$ in a packed vertical column carried out by Lindfors (1979) also showed that density differences as small as 0.02 percent resulted in significantly different RTD's. The importance of these phenomena has recently been revealed in other pieces of chemical apparatus. Holmes et al. (1991) have studied the influence of density gradients on the backmixing coefficient in a reciprocating plate contactor under steady state conditions and Baird et al. (1992) measured unsteady axial mixing by free convection in a vertical empty column. According to the authors, the obtained mixing coefficients are increased significantly by gravitation-driven instabilities as due to vertical concentration gradients. Buoyancy effects on the axial dispersion are also measured in laminar liquid flow through both horizontal and vertical tubes, where significant deviations from the Taylor-Aris dispersion coefficient are observed, see f.i. Reejhsinghani et al. (1966, 1968). Theoretical investigations have been done of buoyancydriven, convective instabilities induced by nonisothermal reactions in porous media with through-flow, see f.i. Gatica et al. (1989) or Nguyen and Balakotaiah (1995).

The major part of experimental research on buoyancy and viscous effects in porous media is dedicated to liquid systems. It is unclear whether differences in the fluid properties have a significant influence on the hydrodynamics of gases flowing through a packed bed, where the role of diffusivity and gravity can be essentially different; experimental data concerning these phenomena in gaseous systems are scarce. Bournia et al. (1961) found deviations from the Taylor-Aris dispersion in laminar flow of gases with the same molecular weight flowing through a vertical empty tube; density differences due to different compressibility factors probably caused the observed deviations, see the estimate of Reejhsinghani et al. (1966). The lack of experimental data, showing an effect of density differences on the RTD in gases flowing through a packed bed, can be explained by the experimental conditions where these data are obtained: almost all experimental programs are performed at ambient pressure, whereas free convection phenomena might be expected to be significant at elevated pressures, where the role of the gravity force is increased at equal Reynolds numbers.

Since a detailed theoretical analysis of the flow behaviour of a gas flowing through complex geometric systems like packed beds is impossible, we have started an extensive experimental program to investigate axial dispersion in the gas phase at conditions more resembling industrial conditions, that is with gradients in the fluid properties and at elevated pressures. Thus, where other authors take measures to suppress the influence of density and viscosity differences between tracer and carrier gas we have introduced them to study their influence on axial dispersion. This can be done by changing the tracer and/or its concentration and/or the total pressure; these measures influence the ratio of different forces governing the flow behaviour. To this end RTD experiments at different tracer concentrations and different pressures are carried out in our laboratory. Experimental results are obtained in an upflow packed column, which can be operated at pressures up to $2.0 \mathrm{MPa}$ and with helium as tracer in a nitrogen flow. The responses to introduced pulse and step changes are measured at two locations in the packed bed and the extent of mixing, represented by the Bodenstein number, is determined by curve fitting in the time domain using the convolution integral and a Gaussian type of transfer function.

The performed pulse experiments show an obvious increase in the dispersion coefficient with an increasing amount of injected tracer and Bodenstein numbers obtained from experiments in which a high density gas is displaced by a low density gas and vice versa, differ as much as a factor ten depending on the density difference and total pressure. It is shown that particularly at elevated pressures, the hydrodynamic behaviour of gases flowing through a packed bed may be significantly influenced by density gradients. In the investigated system, differences in viscosity between tracer and carrier gas seem to have no noticeable influence on the hydrodynamic behaviour. 


\section{EXPERIMENTAL SET-UP AND PROCEDURE}

The experimental set-up is shown schematically in Fig. 1. Two vertical, packed columns with internal diameters of 25 and $50 \mathrm{~mm}$ and a length of $3.7 \mathrm{~m}$ are used. Each column consists of an inlet section of $0.7 \mathrm{~m}$ and a test section of $3.0 \mathrm{~m}$. The pressure in the column can be varied between 0.1 and $2.0 \mathrm{MPa}$ and is controlled with a Back Pressure Regulator placed in the column outlet line. Two bed packings, nonporous spheres with a diameter of 2.2 and $3.9 \mathrm{~mm}$, are used.

Two types of experiments with nitrogen of $99.9 \%$ purity as carrier gas and helium as tracer have been made: pulse injection of helium and step change of the inlet concentration of helium. In the pulse experiments, a small pulse of helium of about $3 \mathrm{mmol}$ is injected via a six port sample valve in the nitrogen flow, which is introduced at the bottom of the column; see Westerterp et al. (1995b) for a more detailed description of the pulse-injection system. Displacement experiments with constant volumetric flow rates are carried out by switching from a small nitrogen to a helium flow - loading - and vice versa - purging - via this sample valve. This flow is fed to the inlet piping; the helium mole fraction in the mixture has been chosen between 0.05 and 0.17 . The helium concentration is measured at the inlet and outlet of the test section by means of thermal conductivity detection cells by taking a small. continuous sample stream of $0-1.5 \mathrm{Nl} / \mathrm{h}$ at these locations. The sample stream is lower than $5 \%$ of the main flow through the packed column; no influence on the experimental results has been observed during the variation of its flow rate and sample point position between the middle and the wall of the column. The detector signal is a linear function of the tracer concentration for helium mole fractions lower than 0.5 . Since the linearity and response time of the analyzers are very important in RTD experiments, the performance of the katharometers has been checked by comparison of the amount of injected helium to the ones determined from the zeroth moment of the peaks: the analyzers work adequately within the experimental range.

The nitrogen flow rate can be controlled from 0 to $5 \mathrm{Nm}^{3} / \mathrm{h}$. The interstitial Reynolds number has been varied in the range 5-250 and the Schmidt number $S c=\eta \rho D_{m o l}=0.23$ is constant and independent of the pressure.

The helium injection system and the conductivity cells are connected to a HP3852A Data Acquisition and Control Unit (DACU). Depending on the expected residence time, the measuring period is set from five minutes to one hour. The control of the tracer injection by the DACU ensures reproducible helium pulses. Since a high measurement resolution is important for a correct determination of the peaks, 1200 data points for time and katharometer signals have been taken, so 600 data points for each peak. The monitored data are stored on the hard disk of a connected computer HP Vectra 486 and processed later.

\section{PARAMETER EVALUATION}

From the data of each experiment the average residence time $\tau$ and the Peclet number $P e=u L \varepsilon D_{a x}$ have to be determined. The raw data are smoothed by means of a mathematical filter, see Golay and Savitzky (1964). After filtering the baseline is reduced to zero by linear regression via the least squares method applied to the data points not belonging to the response signal and the data are converted to RTD curves by normalization.

For the estimation of the parameters $\tau$ and $P e$ different techniques are available, see Wakao and Kaguei (1982). In the past the moment method has often been used although it has the practical disadvantage of overweighing the long tails of the peaks as usually present in experiments. To overcome this problem, the weighted moment method has been developed by Michelsen and Østergaard (1970), where a weight factor is used to decrease the influence of the tail. The problem remains to choose a suitable weighting factor, although Van Gelder and Westerterp (1990) suggested to calculate the weighting factor by minimization of the absolute difference between the experimental response and the theoretical output curves, obtained by calculation of the convolution integral from the experimental input curve and the model transfer function. Nowadays, the most suitable way to obtain estimates for the model parameters is curve fitting in the time domain, since computer calculation times are so short now. Therefore, we used this method and determined $\tau$ and $P e$ directly by minimization of the absolute difference between the experimental and theoretical output curves.

We assume that all mechanisms governing the axial dispersion have characteristic times much lower than the average residence time, so the conditions of randomness of axial tracer velocities are met. In this case, for the calculation of the response output curves the following transfer function can be used:

$$
T R(t)=\sqrt{\frac{P e \tau}{4 \pi t}} \exp \left[-\frac{P e(\tau-t)^{2}}{4 t \tau}\right]
$$

This Gaussian function relates the concentration at one position to that at another location, provided the degree of longitudinal mixing is governed by $P e=u L \varepsilon D_{a r}$. Eq. (1) is the asymptotic solution of the wave model for longitudinal dispersion in the case of an injected unit pulse and for arbitrary initial or boundary conditions, see Westerterp et al. (1995a). Thus in the case of pulse and step change experiments in sufficiently long columns, where concentration fields change slowly, the extent of axial dispersion can be described by only one parameter $P e$. In this region the standard dispersion model is also applicable and its boundary conditions should not influence the results; this was validated for our system by calculations with open and closed boundary conditions. With respect to our 
system, the criteria of Bischoff and Levenspiel (1962) and Han et al. (1985) for applicability of the standard dispersion model for packed beds are also fulfilled.

The following procedure is executed for each experiment:

(a) A first estimation of the model parameters, $\tau_{t}$ and $P e_{\theta}$ is obtained using the method of moments:

$$
\tau_{0}=M_{1, \text { our }}-M_{1, \text { in }} \quad P e_{0}=\frac{2 \tau_{0}^{2}}{M_{2, \text { out }}-M_{2, \text { in }}-M_{1, \text { out }}{ }^{2}+M_{1, \text { in }}{ }^{2}}
$$

where $M_{1}$ and $M_{2}$ are, respectively, the first and second time moments of the normalized RTD curves, defined by

$$
M_{k}=\int_{0}^{\infty} Y(t) t^{k} d t
$$

in the case of pulse experiments and by

$$
M_{k}=\int_{0}^{\infty}\left|\frac{d Y(t)}{d t}\right| t^{k} d t
$$

for displacement experiments, where $Y$ is the normalized mole fraction of helium.

(b) The theoretical response curve is calculated with the convolution integral

$$
E_{c o n v}(t)=\int_{0}^{t} \operatorname{TR}(p) Y_{\text {in }}(t-p) d p
$$

where $Y_{i n}$ is the normalized experimental input curve and $T R(t)$ the transfer function given by eq. (1).

The difference between the experimental and theoretical output response curves defined by:

$$
\Delta A=\int_{0}^{\infty}\left|E_{\text {conv }}(t)-Y_{\text {out }}(t)\right| d t
$$

is minimized using a Nelder Mead optimization method. This target function assigns importance especially to the middle of the peak and not so to the tails where the experimental error is relatively large.

(d) The desired Bodenstein and Reynolds numbers are now obtained using the fitted model parameters $\tau$ and $P e$, the geometrical data of the test system and the physical properties of pure nitrogen.

In the case of pulse experiments, the accuracy of the calculated parameters can be characterized by the absolute difference of the experimental and theoretical output curves as defined in eq. (5). The average value of $\triangle A$ is 0.04 with a standard deviation of 0.02 , where the area under both the experimental and the theoretical output response curves is equal to one. These low values of $\Delta A$ lead to the conclusion that the mixing in the pulse experiments is described adequately by the Gaussian transfer function, eq. (1). In the case of the displacement experiments, the accuracy of the calculated response output curves to describe the experimentally obtained curves is of the same order as compared to the pulse experiments.

\section{RESULTS}

Measurements of the residence time distribution have been carried out at pressures up to $1.5 \mathrm{MPa}$ and at interstitial Reynolds numbers between 5 and 250. First we will present our pulse experiments and afterwards step change experiments from high to low densities and vice versa at different pressures. All experiments are performed with an upflow of the gas through the packed bed.

\section{Pulse experiments at elevated pressure}

Bodenstein numbers obtained at different pressures are plotted versus the interstitial Reynolds number in Fig. 2. The experiments show there is an unexpected difference in the Bodenstein numbers at different pressures and Reynolds numbers above 10. At low flow rates the dispersion is dominated by molecular diffusion and the different lines are converging as expected because the Schmidt number is independent of pressure in the investigated 


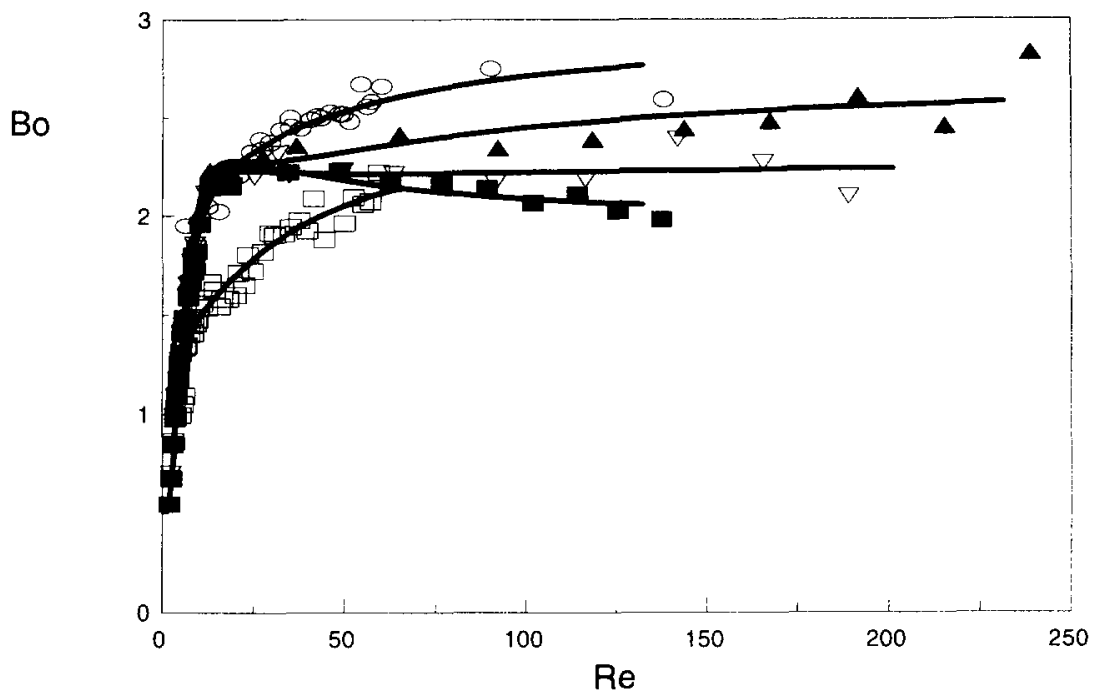

Fig. 2. Experimental Bodenstein numbers obtained from pulse experiments at different column pressures; $d_{1}=50 \mathrm{~mm}$, $d_{p}=2.2 \mathrm{~mm}, L=3.0 \mathrm{~m}$. $0.2 \mathrm{MPa}: \nabla 0.3 \mathrm{MPa} \approx 0.5 \mathrm{MPa} ; \circ 1.0 \mathrm{MPa} ; \square 1.5 \mathrm{MPa}$.

experimental range. At these flow rates, the axial dispersion coefficient is related to the molecular diffusivity by $D_{a x}=\gamma D_{m e l}$ where $\gamma$ is the reciprocal tortuosity factor of the packing determined to be 0.78 which is in accordance with factors found in literature. Increasing the pressure up to $1.0 \mathrm{MPa}$ we observe a slight increase in the Bodenstein numbers at higher Reynolds numbers, whereas at 1.5 MPa the Bodenstein numbers are significant smaller than those at lower pressures. The latter effect may be a result of an increase in the amount of injected helium at $1.5 \mathrm{MPa}$ from 3 to $6 \mathrm{mmol}$. At higher pressures, the tracer initially occupies a smaller volume, whereas the width of the pulse at a sufficiently long distance from the injection point is approximately the same. As a consequence, the helium fraction at the detection points decreases resulting in a smaller peak/noise ratio. To suppress the experimental error due to low peak/noise ratios more helium has to be injected. To validate these observations the influence of the amount of tracer in pulse experiments has to be investigated.

Influence of the amount of tracer

Pulse experiments have been carried out with different amounts of helium between 2 and $7 \mathrm{mmol}$; the obtained Bodenstein numbers are presented in Fig. 3. The results show an evident increase of axial dispersion with an increase in the amount of injected tracer; this may be caused by differences in the fluid properties. An increase of tracer

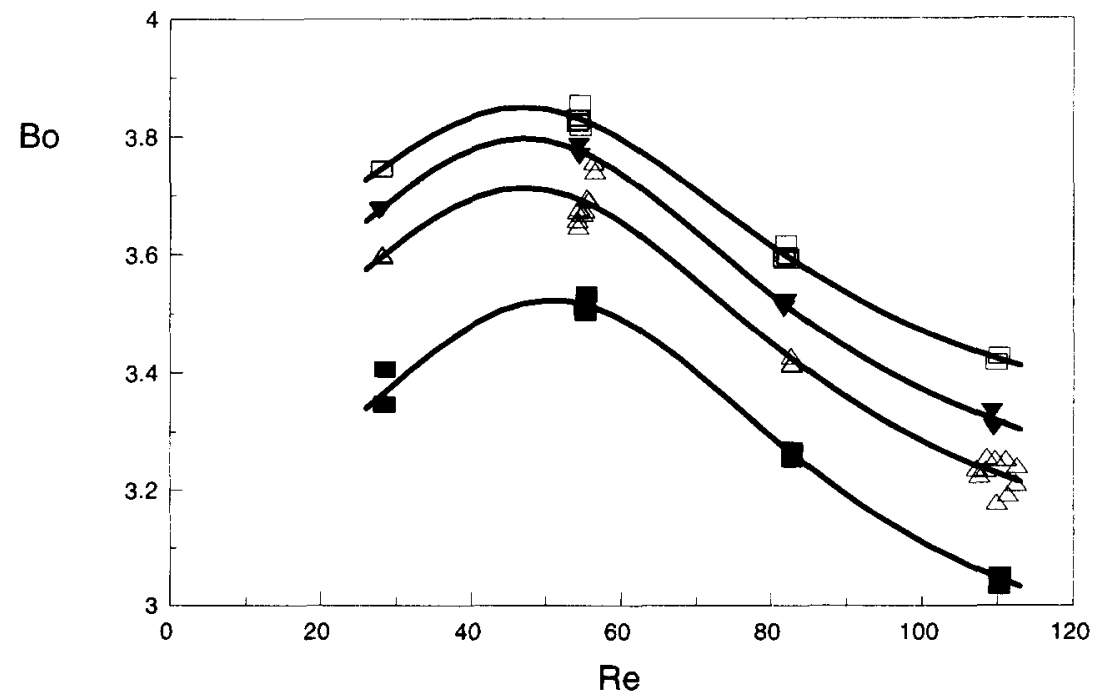

Fig. 3. Influence of the amount of injected helium on the calculated Bodenstein number in pulse experiments obtained at $0.5 \mathrm{MPa} ; d_{i}=50 \mathrm{~mm}, d_{p}=2.2 \mathrm{~mm}, L=3.0 \mathrm{~m}$. $\square 2 \mathrm{mmol} ; 3 \mathrm{mmol} ; \Delta 5 \mathrm{mmol} ; 7 \mathrm{mmol}$ 


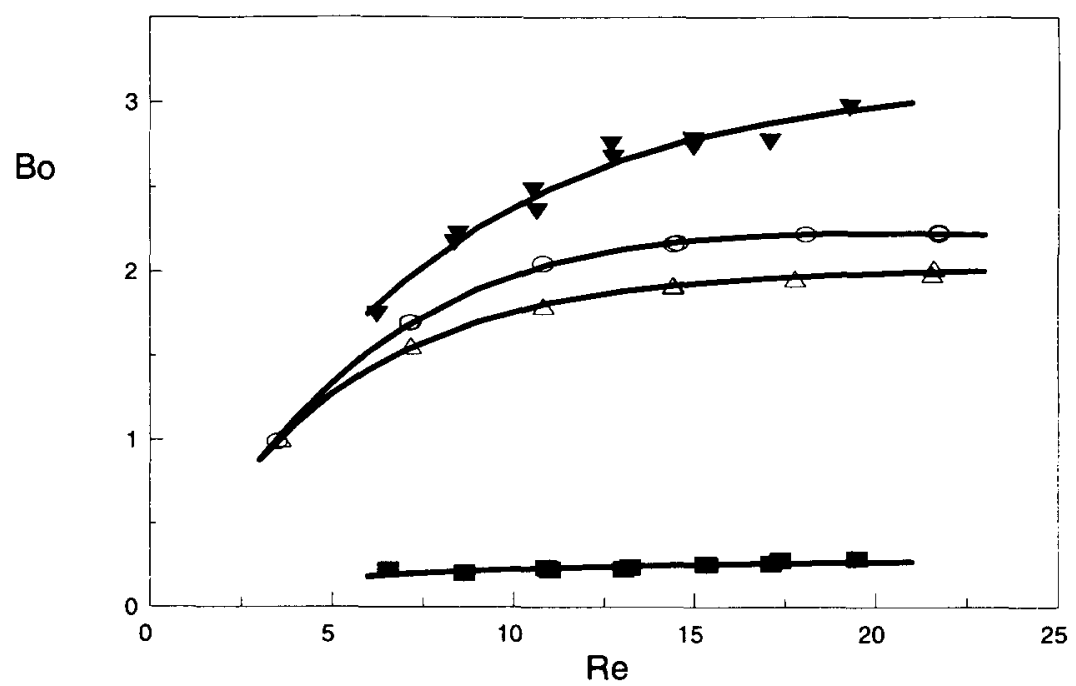

Fig. 4. Relationship between the Bodenstein number and Reynolds number for step changes from pure nitrogen to a helium/nitrogen mixture and vice versa at 0.5 and $1.5 \mathrm{MPa} ; \Delta \rho^{*}=14 \%$ and $\Delta \eta^{\circ}=-3 \%, d_{1}=50 \mathrm{~mm}, d_{p}=2.2 \mathrm{~mm}$, $L=3.0 \mathrm{~m}$. loading, $1.5 \mathrm{MPa}$; purging, $1.5 \mathrm{MPa} ; \Delta$ loading, $0.5 \mathrm{MPa}$; o purging $0.5 \mathrm{MPa}$.

material leads to larger density and viscosity differences, evidently resulting in a faster spreading of the helium peak in the nitrogen flow. Although it must be theoretically possible to approach an amount of helium where there is no noticable influence of the tracer anymore, such low concentrations cannot be detected by our analytical instruments.

\section{Displacement experiments}

Free convection phenomena are investigated by performing step change experiments with loading and purging of helium into nitrogen in an upflow direction. The results presented in Fig. 4 show at two different column pressures a significant difference in Bodenstein numbers obtained by loading or purging. The loading experiments, where pure nitrogen is displaced by the helium/nitrogen mixture, show more axial mixing than the purging experiments, particularly at higher pressures. In Fig. 4 the Bodenstein numbers at $1.5 \mathrm{MPa}$ differ approximately a factor ten, while the density difference is only $14 \%$ and the viscosity difference $3 \%$.

Loading and purging experiments at higher Reynolds numbers are presented in Fig. 5. It was expected that the influence of differences in density and viscosity on the axial mixing should decrease. However, the experiments at 1.5 $\mathrm{MPa}$ indicate at higher Reynolds numbers an unexpected reversing of the difference in the extent of axial mixing

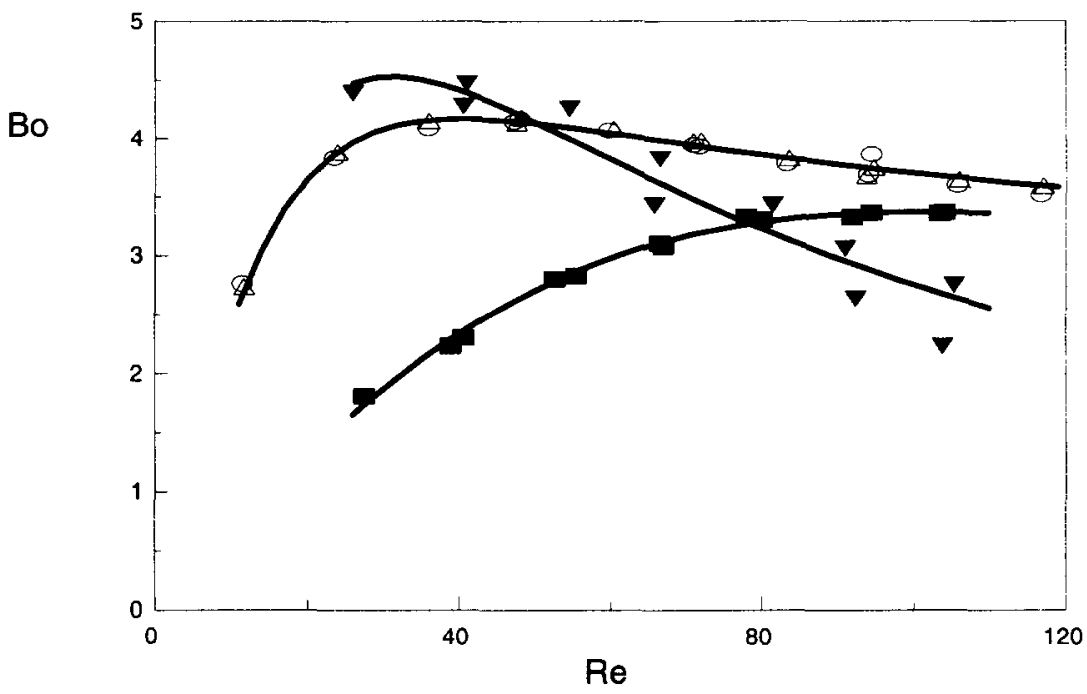

Fig. 5. Relationship between the Bodenstein number and Reynolds number for positive and negative step changes at conditions where the influence of differences in fluid properties is almost negligible and highly significant; $d_{1}=25 \mathrm{~mm}$, $d_{p}=3.9 \mathrm{~mm}, L=3.0 \mathrm{~m}$.

- loading, $\Delta \rho^{*}=14 \%$ and $\Delta \eta^{*}=-3 \%, 1.5 \mathrm{MPa}$; purging, $\Delta \rho^{*}=14 \%$ and $\Delta \eta^{*}=-3 \%, 1.5 \mathrm{MPa}$ $\Delta$ loading, $\Delta \rho^{*}=4 \%$ and $\Delta \eta^{*}=-0.7 \%, 0.5 \mathrm{MPa} ; \circ$ purging, $\Delta \rho^{*}=4 \%$ and $\Delta \eta^{*}=-0.7 \%, 0.5 \mathrm{MPa}$ 
for loading and purging of helium. For a full verification of this observation additional experiments at even higher flow rates are needed. Also shown in Fig. 5 are the results obtained with small differences in density and viscosity at $0.5 \mathrm{MPa}$. Under these conditions the Bodenstein numbers for loading and purging experiments are equal and now may represent the "true" axial mixing of an uniform gas flowing through a packed bed.

\section{DISCUSSION AND CONCLUSIONS}

It is well known that mixing in the longitudinal direction in packed beds occurs through a combined action of molecular diffusion and hydrodynamical mixing. Usually the fluid flow is considered to be independent of variations of the fluid properties and often measures are taken to exclude the influence of free convection. However, available experimental data on dispersion in liquids, see f.i. Hill (1952) and Lindfors (1979), show that both small density and viscosity variations may be important factors influencing mixing in packed beds. These results are in agreement with available theoretical considerations, see Nunge and Gill (1969) and Homsy (1987). In this case, the practical significance of the obtained results is questionable. Experimental data on the influence of variable fluid properties on the flow of gases are scarce; higher values of the dispersion coefficient in short beds obtained by tracer experiments, see f.i. Langer et al. (1978), give rise to a discussion of free convection phenomena in gases flowing through a packed bed. The unexplained experimental results of Chao and Hoelscher (1966) and Kehinde et al. (1983) on axial dispersion under conditions of interphase mass transfer, where the properties of the gas are changed during the dispersion experiments, perhaps also relate to the mentioned problem. The experimental results as presented in Fig. 2-5 show that variable fluid properties can have a significant influence on the flow of gases through a packed bed. Moreover, pulse experiments carried out after shortening of the test section to $2.0 \mathrm{~m}$ by changing the position of the second detection point confirm these results: lower Bodenstein numbers are obtained compared to those for the $3.0 \mathrm{~m}$ test section, with otherwise constant conditions. Since the dispersion in packed beds involves some rather complex phenomena, we are only in the stage of an approximate analysis of the problem and additional experiments are desirable with different tracers and/or with downflow of the gas. We will report on further experiments in near future and confine us here to a short qualitative discussion of our results.

Our experiments exhibit an essential influence on the extent of axial dispersion of the amount of tracer, of the total pressure and of the direction of change in fluid properties in the displacement experiments. The influence of these factors may all be attributed to the occurrence of free convection and the change of the ratio of buoyancy force and hydrodynamic resistance. The strong dependence of mixing on total pressure is a strong confirmation of this, because at equal initial conditions and for the same Reynolds and Schmidt numbers, the ratio of the buoyancy force to viscous and inertial forces is proportional to $P^{2}$. Similar observations are done by Katto and Masuoko (1967) in the case of an initially stagnant fluid; they found experimentally that convective flow in a porous medium under a reasonable temperature gradient can be generated by compressing the gas. Both variations in density and viscosity may be responsible for the generation and intensity of free convection. According to Hill's approach (1952), in our experiments the influence of the viscosity difference on the flow stability is opposite to that of gravity, because the viscosity of the helium/nitrogen mixture is higher than of pure nitrogen. However, an estimate of the instability conditions shows - when inertial forces are taken into account - that the viscosity difference between the two fluids is hardly important in our case. Thus, the observed differences in mixing evidently must be ascribed to density differences between the helium/nitrogen mixture and pure nitrogen. A change in density occurs also due to the pressure drop across the column, although in our experiments these changes are negligible compared to those due to different gas compositions, particularly at elevated pressures.

In the displacement experiments, an increase in pressure results in an increase in axial dispersion if the lighter gas displaces the heavier gas and in a decrease when the heavier gas displaces the lighter, see Fig. 4. The Bodenstein numbers for pulse experiments at equal pressures, presented in Fig. 2, lie in between the data for positive and negative density step changes of Fig. 4. This also indicates that buoyancy forces due to density variations are more significant than those due to viscosity changes in our experiments, otherwise we would expect an opposite trend.

An interesting effect is observed when $R e$ increases at $1.5 \mathrm{MPa}$. see Fig. 5. In the case of displacement experiments, where the heavier gas displaces the lighter one, an increase of the pressure results in a decrease of axial dispersion at lower Reynolds numbers, whereas at higher Reynolds numbers an opposite effect is observed. Here it must be noted that buoyancy forces may have a twofold effect on axial dispersion by influencing both the velocity nonuniformity and the intensity of radial mixing. Reejhsinghani et al. (1966) also found for laminar flow in an empty, horizontal tube that free convection can both enhance and suppress dispersion significantly by increasing the flow nonuniformity and the radial mixing, respectively.

We should realize that the used transfer function, eq. (1), seems to be less adequate to describe the displacement experiments where the intensity of mixing is increased significantly due to free convection. During these experiments, the concentration fields are changing so rapidly that the transfer function can not describe the mixing accurately, although the calculated Bodenstein numbers may be considered as a good indication of the extent of mixing.

In our experimental work, the main attention has been paid to measurements at elevated pressures. Such experiments are of interest because they help us to elucidate the role of different factors influencing the gas flow through a packed bed and because of practical reasons: many processes proceed at elevated pressures or at variable pressures like in Pressure Swing Adsorption. Experimental data on gas dispersion in packed beds at elevated 
pressures are almost absent. In this context, the work of Sarmah and Haynes (1989) should be mentioned. The authors made pulse experiments at elevated pressures to measure the effective diffusivity in porous catalysts. They did not observe an influence of the amount of methane injected into a helium stream on the response curves and proposed to use elevated pressures to reduce the axial dispersion contribution and at the same time to increase the contribution due to intraparticle diffusion. Their pulse dispersion experiments were performed with dilute mixtures of methane and helium; the maximum concentration of methane did not exceed 2.8 mole $\%$. Our experiments show that a pressure increase may essentially influence axial dispersion - at least when the concentration of the tracer is not negligible small - and that care should be taken when the procedure of Sarmah and Haynes (1989) is used

Probably the theory of flow behaviour in packed beds will never reach a predictive level and will always remain closely linked to experimental programs for parameter evaluation. To improve the present situation, the need for physically more realistic models is clear. However, we must realize lumped model parameters cover many different mechanisms and by changing the experimental conditions the relative influence of these mechanisms may change 10o. Therefore it is risky to determine lumped model parameters at experimental conditions not corresponding to the practical situation. This study demonstrates a significant influence of pressure on the axial dispersion if density differences between the injected tracer and the carrier gas exist. In this case, the movement of the tracer does not represent the flow characteristics of the carrier gas and the practical relevance of the obtained axial dispersion data might be questionable. A not justified neglect of the influence of the tracer on the behaviour of fluids flowing through a packed bed might be one of the reasons of the large scatter in the experimental data found in literature.

In our experiments, the transition region between the two fluids flowing through the column has a length of 0.2-1 $\mathrm{m}$, depending on the axial coordinate and the Bodenstein number. Density differences as used in our study over such lengths, are certainly not unusual in chemical apparatus where concentration and temperature gradients occur due to interphase heat and mass transfer or chemical reaction. In short columns with a low value of the Peclet number, as in pilot plants, in laboratories or in off-gas treating units, free convection phenomena may have a tremendous influence on the performance of the column and might be one of the causes of the problems in the mathematical modelling of such systems.

Acknowledgement - We owe very much to J. Nies and M.H.A. Rolink, who took part in the experimental program. We further wish to express our appreciation to G.H. Banis, F. ter Borg, K. van Bree, O.D. Veehof and A.H. Pleiter for their technical assistance.

\section{NOTATION}

$d_{p} \quad$ particle diameter, $\mathrm{m}$

$d_{t} \quad$ tube diameter, $\mathrm{m}$

$D_{u x} \quad$ axial dispersion coefficient, $\mathrm{m}^{2} / \mathrm{s}$

$D_{m e t} \quad$ molecular diffusion coefficient, $\mathrm{m}^{2} / \mathrm{s}$

$L \quad$ length of the test section, $m$

$M_{i} \quad i$ th moment of the normalized RTD curve, $\mathrm{s}^{\prime}$

$P \quad$ pressure, $\mathrm{MPa}$

$u \quad$ superficial fluid velocity averaged over cross section, $\mathrm{m} / \mathrm{s}$

$Y \quad$ normalized helium mole fraction

\section{Greek letters}

$\Delta \rho^{*} \quad$ dimensionless density difference $\left(=\left[\rho_{N 2}-\rho_{H_{e} N 2 \text {-mikture }}\right] / \rho_{N 2} * 100 \%\right)$

$\Delta \eta^{*} \quad$ dimensionless viscosity difference $\left.\left(=/ \eta_{N 2}-\eta_{H e N 2 \text {-nnixure }}\right] / \eta_{N 2} * 100 \%\right)$

$\varepsilon \quad$ bed porosity

$\eta \quad$ dynamic viscosity, Pa s

$\rho$ density, $\mathrm{kg} / \mathrm{m}^{3}$

$\tau \quad$ average residence time, $s$

\section{Dimensionless groups}

Bo Bodenstein number for axial dispersion ( $\left.=u d_{d} / \varepsilon D_{a x}\right)$

$B o_{m o l} \quad$ Bodenstein number for molecular diffusion $\left(=u d_{j} / \varepsilon D_{m o l}\right)$

$P e \quad$ Peclet number for axial dispersion $\left(=u L \varepsilon D_{u x}\right)$

Re interstitial Reynolds number $\left(=\rho_{N_{2}} u d_{1 /} / \varepsilon \eta_{N 2}\right)$

Sc Schmidt number $\left(=\eta_{N 2} / \rho_{N 2} D_{m o t}\right)$

\section{REFERENCES}

Baird, M.H.I., Aravamudan, K., Rama Rao, N.V., Chadam, J. and Peirce, A.P., 1992, Unsteady axial mixing by natural convection in a vertical column. AlChE J. 38, 1825-1834.

Bischoff, K.B. and Levenspiel, O., 1962, Fluid dispersion - generalization and comparison of mathematical models - Il Comparison of models. Chem. Engng Sci. 17, 257-264.

Bournia, A., Coull, J. and Houghton, G., 1961, Dispersion of gases in laminar flow through a circular tube, Proc. 
Roy. Soc. London A261, 227-236.

Chao, R. and Hoelscher, H.E., 1966, Simultaneous axial dispersion and adsorption in a packed bed. AIChE J. 12, 271-278

Gatica, J.E., Viljoen, H.J. and Hlavacek, V., 1989, Interaction between chemical reaction and natural convection in porous media. Chem. Engng Sci. 44, 1853-1870.

Golay, M.J.E. and Savitzky, A., 1964, Smoothing and differentiation of data by simplified least square procedures. Analytical Chemistry 36, 1627-1639.

Gunn, D.J. and Pryce, C., 1969, Dispersion in packed beds. Trans. Instn. Chemt. Engrs. 47, 341-350.

Gunn, D.J., 1969, Theory of axial and radial dispersion in packed beds. Trans. Instn. Chem. Engrs. 47, $351-359$.

Gunn, D.J., 1993, On axial dispersion in fixed beds. Chem. Eng. Process. 32, 333- 338.

Han, N.W., Bhakta, J. and Carbonell, R.G., 1985, Longitudinal and lateral dispersion in packed beds: Effect of column length and particle size distribution. AIChE J. 31, 277-288.

Hiby, J.W., 1962, Longitudinal and transverse mixing during single-phase flow through granular beds. In Symp. on the interaction between fluids and particles, Instn. of Chem. Engrs., London, 312-325.

Hill, S., 1952, Channelling in packed columns. Chem. Engng Sci. 1, 247-253.

Holmes, T.L., Karr, A.E. and Baird, M.H.I., 1991, Effect of unfavorable continuous phase density gradient on axial mixing. AlChE J. 37, 360-366.

Homsy, G.M., 1987, Viscous fingering in porous media. Ann. Rev. Fluid Mech. 19, 271-311

Katto, Y. and Masuoko, T., 1967, Criterion for the onset of convective flow in a fluid in a porous medium. Int. J. Heat Mass Transfer 10, 297-309.

Kehinde, A.J., Hudgins, R.R. and Silveston, P.L., 1983, Measurement of axial dispersion in packed beds at low Reynolds numbers by imperfect pulse chromatography. J. Chem. Eng. Japan 16, 476-482.

Langer, G., Roethe, A., Roethe, K.-P. and Gelbin, D., 1978, Heat and mass transfer in packed beds - III. Axial mass dispersion. Int. J. Heat Mass Transfer 21, 751-759.

Levich, V.G., Markin, V.S. and Chismadzhev, Yu.A., 1967, On hydrodynamic mixing in a model of a porous medium with stagnant zones. Chem. Engng Sci. 22, 1357-1367.

Lindfors, L.-E., 1979, Influence of small density differences on axial dispersion in single-phase liquid flow through spherical packings. Chem. Eng. J. 18, 257-261.

Michelsen, M.L. and Østergaard, K., 1970, The use of residence time distribution data for estimation of parameters in the axial dispersion model. Chem. Engng Sci. 25, 583-592.

Moulijn, J.A. and Van Swaaij, W.P.M., 1976, The correlation of axial dispersion data for beds of small particles. Chem. Engng Sci. 31, 845-847.

Nguyen, D and Balakotaiah, V., 1995, Reaction-driven instabilities in downflow packed beds. Proc. Roy. Soc. London A450, $1-21$.

Nunge, R.J. and Gill, W.N., 1969, Mechanisms affecting dispersion and miscible displacement. Ind. Eng. Chem. 61, 33-49.

Reejhsinghani, N.S., Gill, W.N. and Barduhn, A.J., 1966, Laminar dispersion in capillaries: Part III. Experiments in horizontal tubes including observations on natural convection effects. AlChE J. 12, 916-921.

Reejhsinghani, N.S., Barduhn, A.J. and Gill, W.N, 1968, Laminar dispersion in capillaries: Part V. Experiments on combined natural and forced convection in vertical tubes. AlChE J. 14, 100-109.

Sarmah, S.K. and Haynes, H.W., 1989, Effective diffusivity by pulse gas chromatography at elevated pressures. AlChE J. 35, 1728-1734.

Tsotsas, E. and Schlünder, E.U., 1988, On axial dispersion in packed beds with fluid flow. Chem. Eng. Process. 24, $15-31$.

Tsotsas, E. and Schlünder, E.U., 1994, Comments on studies on axial dispersion in fixed beds. Chem. Eng. Process. 33, $107-111$.

Van Gelder, K.B. and Westerterp, K.R., 1990, Residence time distributions and hold-up in a cocurrent upflow packed bed reactor at elevated pressure. Chem. Eng. Technol. 13, 27-40.

Wakao, N and Kaguei, S., 1982, Heat and mass transfer in packed beds (Topics in Chemical Engineering; v. 1), Gordon and Breach Science Publishers, New York.

Westerterp, K.R. and Wijngaarden, R.J., 1990, Dispersion in packed beds: does the fluid elasticity play a role? Dev. in Chem. Eng. 14, 117-128.

Westerterp, K.R., Dil'man, V.V., Kronberg, A.E. and Benneker, A.H., 1995a, Wave model for longitudinal dispersion: analysis and applications. AIChE J. 41, 2029-2039.

Westerterp, K.R., Wijngaarden, R.J. and Nijhuis, N.B.G., 1995b, Does the fluid elasticity influence the dispersion in packed beds? Chem. Eng. Technol. (accepted for publication). 